\title{
Parental perception of weight status: Influence on children's diet in the Gateshead Millennium Study
}

\author{
S. Almoosawi ${ }^{1}$, A. R. Jones ${ }^{1}$, K. N. Parkinson ${ }^{1}$, M. S. Pearce ${ }^{2}$, H. Collins ${ }^{3}$, A. J. Adamson ${ }^{1}$ \\ and the Gateshead Millennium Study core team \\ ${ }^{1}$ Institute of Health \& Society and Human Nutrition Research Centre, Newcastle University, Newcastle upon Tyne, \\ NE2 4HH, ${ }^{2}$ Institute of Health and Society and Sir James Spence Institute of Child Health, Royal Victoria Infirmary, \\ Newcastle upon Tyne, NE2 4HH and ${ }^{3}$ Faculty of Medical Sciences, Newcastle University, Newcastle upon Tyne, \\ NE2 $4 H H$
}

Childhood overweight and obesity is a key public health priority ${ }^{(1)}$. It is increasingly recognised that parents' perceptions of their child's weight status is an important issue when planning public health interventions to reduce the prevalence of overweight and obesity. However, few studies have examined the association between parents' perceptions of their child's weight status and their children's diet. The present study examined the association between parental perceptions of child weight status and child's diet.

Participants were members of the Gateshead Millennium Study ${ }^{(2)}$. Parental perception of their child's weight status was assessed using a questionnaire and compared against International Obesity Task Force cut-offs for childhood overweight and obesity ${ }^{(3)}$ when the children were aged 6-8 years old. Diet was assessed at age 6-8years old using the FAST food diary method ${ }^{(4)}$. Dietary patterns were identified using principal component analysis. The association between parental perception and dietary patterns was assessed using multivariate regression after adjustment for child's gender, child's weight status, maternal body mass index (BMI), maternal education and depivation status.

Of the 361 parents who provided data on their perception of their child's weight status and who had complete data on confounders and child's diet, $63(17 \%)$ parents perceived their child's as being of 'normal' weight or 'overweight' when they were actually 'overweight' or 'obese', respectively. No parent overestimated their child's weight status. Parents who did not correctly perceive their child's weight status were more likely to be overweight or obese $(68.3 \%$ vs. $45.0 \%$ of parents who perceived their child's weight correctly; $P$-value $=0.002)$, and have an overweight or obese child $(100.0 \%$ vs. $6.4 \%$ of parents who perceived their child's weight correctly; $P$-value $<0.001)$.

Overall, four dietary patterns were derived accounting for $22.5 \%$ of variance of food intake. The first dietary pattern accounted for the largest proportion of variation in food intake (6.7\%). This dietary pattern resembled a 'healthy' dietary pattern and had positive correlations with vegetables and whole-grain cereal intake, and negative correlations with refined cereals and chips. After adjustment for confounders, parents who did not perceive their child's weight correctly had children with a lower 'healthy' dietary pattern score compared to children whose parents correctly perceived their weight $(\beta=-0 \cdot 88 ; 95 \% \mathrm{CI}:-1 \cdot 7,-0 \cdot 1 ; P$-value $=0 \cdot 028)$. This association was found despite a higher proportion of reduced-sugar carbonated drinks consumers amongst children whose parents incorrectly perceived their weight status compared to children whose parents perceived their weight correctly $(52 \cdot 4 \%$ vs. $33 \cdot 6 \% ; P$-value $=$ 0.005). No associations between parental perception and other dietary patterns were observed.

In conclusion, parental perception is an important predictor of children's diet. Parents who did not correctly perceive their child's weight status had children who scored lower on the 'healthy' dietary pattern, despite higher consumption of diet products such as reduced sugar carbonated drinks. These findings suggest parental perception as an important target for intervention. Further research is required to define parents' diets based on their perception status and to examine if a child's or parent's diet mediates the association between parental perception and child weight.

1. World Health Organisation. (2014) http://www.who.int/dietphysicalactivity/childhood/en/

2. Parkinson KN, Pearce MS, Dale A, et al. (2011) Int J Epidemiol 40, 308-317.

3. Cole TJ, Bellizzi MC, Flegal KM, et al. (2000) Brit Med J 320, 1240-1243.

4. Adamson AJ, Griffiths JM, Carlin LE, et al. (2003) Proc Nutr Soc 62, 84A. 